\title{
Répercussions de la COVID-19 sur l'incidence de blessures graves causées par la violence intentionnelle, les traumas accidentels et des traumas découlant d'une tentative de suicide au Canada
}

Les opinions exprimées dans cet éditorial sont celles de l'auteur et ne représentent pas nécessairement celles de l'Association médicale canadienne ou ses filiales.

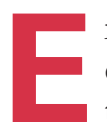
n mars 2020, la vie en Amérique du Nord a changé du tout au tout après l'introduction sur le territoire de la maladie à coronavirus 2019 (COVID19), causant une pandémie d'une intensité jamais vue depuis plusieurs générations. La confusion, la désinformation et la peur généralisée dominaient nos pensées alors que nous nous précipitions vers nos hôpitaux d'attache, prêts à adopter de nouvelles façons de vivre et de travailler - une révolution qu'aucun d'entre nous n'aurait pu prédire. Durant l'année qui a suivi, nos vies ont connu des bouleversements sur les plans scientifique, économique, social, émotionnel et professionnel, ce qui pourrait changer nos vies de façon permanente, de manières positives et négatives.

Les retombées directement attribuables à la COVID19 sur des aspects de la santé publique touchant chacun de nos services de chirurgie continuent d'être bien documentées et d'évoluer ${ }^{1}$. Une surspécialité au carrefour de plusieurs de ces aspects (médical, chirurgical, social, scientifique) est la traumatologie, et donc par extension les services de chirurgie et de traumatologie. Bien que les intervenants en traumatologie du Canada aient régulièrement des discussions anecdotiques animées et intéressantes sur le sujet, peu de données concrètes ont été publiées à ce jour. Nos collègues des États-Unis ont récemment présenté une étude de cas pertinente (suivie d'un commentaire) portant sur l'incidence de la COVID19 sur les caractéristiques épidémiologiques locales des traumas. Plus précisément, ils ont observé une augmentation des blessures intentionnelles (particulièrement par armes à feu) alors que les visites aux urgences et les traumas accidentels étaient en baisse ${ }^{2}$. Notons ici que l'interprétation des auteurs demeure controversée (politiques d'isolement social sans précédent c. d'autres théories comme les ventes records d'armes à feu aux États-Unis). Cela dit, ces conclusions sont particulièrement intrigantes, surtout si on considère qu'habituellement, la plupart des décès par arme à feu $(66 \%)$ sont dus à un suicide plutôt qu'à un homicide.
Malheureusement, nous n'avons pu trouver de données sur le suicide dans la littérature publiée à ce jour.

Afin d'évaluer l'incidence des comportements sociaux, des réalités économiques et des politiques de santé publique en contexte de pandémie sur les blessures graves, nous avons fait des recherches dans le registre de traumatologie du Centre médical Foothills, à Calgary (Canada), en visant les 3 mois suivant la déclaration d'une urgence de santé publique en Alberta (le 15 mars 2020). Pour tenir compte dans la comparaison du risque de biais découlant de la répartition saisonnière des blessures, les patients retenus $(n=357)$ ont été appariés selon la date à des patients gravement blessés d'une cohorte correspondant au même intervalle saisonnier de l'année précédente (après confirmation que cette année était typique d'une distribution normale de patients établie sur 5 ans) $)^{3}$. Notons que l'âge médian des patients (50,1 ans c. 52,3 ans, $p>0,05)$, l'indice de gravité des blessures (IGB; 19 c. $18, p>0,05$ ) et le pourcentage d'instabilité hémodynamique à l'admission sont demeurés stables, mais que le nombre de femmes gravement blessées a connu une baisse dramatique $(29,1 \%$ c. $21,0 \%, p=0,012)$. Ces observations ont été faites dans un contexte où le nombre total de visites aux urgences était en baisse par rapport à l'année précédente, alors que le nombre d'hospitalisations pour polytraumatisme grave était demeuré similaire pour la même période (373 c. 357 ). Bien que le nombre total de blessures graves découlant d'accidents de la route et d'actes de violence intentionnels (traumatismes contondants, agressions à l'arme blanche, blessures par balle: $21,3 \%$ c. $22,7 \%, p=0,651$ ) soit demeuré stable, la nature du mécanisme des blessures a changé substantiellement. Ainsi, la proportion de traumatismes contondants a connu une forte baisse $(7,3 \% \mathrm{c}$. $3,4 \%, p=0,020)$, mais celle des traumas pénétrants a augmenté $(14,0 \%$ c. $19,6 \%, p=0,045)$. Par ailleurs, l'augmentation des traumas pénétrants était due à une augmentation uniforme des blessures par balle $(16,0 \%$ des traumas pénétrants c. $32,9 \%, p=0,006)$. Puisque nous n'avons pas observé d'augmentation massive des 
acquisitions de nouvelles armes de poing durant la COVID-19 dans la population canadienne, comme ce fut le cas aux États-Unis, cette observation donne à réfléchir.

L'association entre le suicide et l'utilisation d'armes à feu à mauvais escient a été établie depuis des décennies. Il est donc intéressant de se pencher sur la stabilité du nombre de tentatives de suicide causant des blessures graves durant la phase initiale de la pandémie de COVID-19 $(4,8 \%$ c. $6,2 \%, p=0,410)$. Notons toutefois que ces données n'incluent pas les décès par suicide avant l'arrivée à l'hôpital, et qu'elles pourraient bien sûr continuer d'évoluer au fil du temps, alors que l'accumulation du stress social et financier découlant du confinement prolongé imposé par les autorités de santé publique affectera certains de nos citoyens les plus vulnérables. Si on se penche plus particulièrement sur la violence et les tentatives de suicide touchant les femmes, on constate clairement qu'alors que le nombre de femmes gravement blessées à la suite d'une agression, que ce soit par traumatisme contondant, agression à l'arme blanche ou blessure par balle, a connu une baisse importante $(5,6 \%$ c. $0,84 \%, p=$ $0,003)$, le nombre de blessures graves découlant d'une tentative de suicide est quant à lui demeuré inchangé. De plus, si on analyse séparément les mécanismes des blessures subies par cette population, on peut voir que les traumatismes contondants $(2,0 \%$ c. $0,2 \%, p=0,033)$ et pénétrants $(3,6 \%$ c. $0,80 \%, p=0,012)$ ont tous deux connu une baisse. C'est la première fois que ces observations cruciales ont été dégagées de données relatives au contexte de la COVID-19. Chacune des nombreuses hypothèses qu'on peut formuler sur l'évolution des mécanismes mérite une réflexion approfondie et d'autres études à l'échelle nationale. Par exemple, bien qu'on puisse trouver rassurante la baisse du nombre de patientes présentant des blessures graves résultant de la violence familiale (traumatismes contondants et pénétrants), il se pourrait que la fréquence d'incidents de violence familiale causant des blessures qui n'atteignent pas ce seuil ait connu une hausse substantielle (c.-à-d. qu'il pourrait y avoir une baisse du nombre de patientes se présentant aux urgences avec des blessures «mineures» en raison des politiques de confinement). Et n'oublions pas que les chiffres présentés ici n'incluent que les patients gravement blessés (IGB $\geq 12$ ) qui arrivent vivants à l'hôpital. En d'autres mots, ils excluent les personnes présentant des blessures mineures, qui sont évaluées par nos collègues urgentologues non spécialisés en traumatologie. Par ailleurs, on pourrait penser que la stabilité apparente de la mortalité globale des patients gravement blessés est rassurante, mais les données analysées n'incluent pas les personnes décédées avant leur arrivée au centre de traumatologie, quel que soit le contexte (mécanismes intentionnels ou accidentels).

Il est devenu évident, en cette ère moderne de crises de santé publique, que la COVID-19 a poussé à sa limite notre capacité à prédire, à traiter et à gérer des change- ments importants touchant le bien-être de notre société. Si nous voulons mieux nous préparer à la prochaine pandémie ou à la prochaine urgence médicale, nous devrons nous donner la capacité de procéder à une analyse détaillée des données à l'échelle du pays qui soit indépendante des conclusions de nos voisins du Sud (ou de tout autre pays) et des sources de biais potentielles qui ont marqué les époques précédentes. Heureusement, le groupe CANUCS a déjà commencé à se pencher sur les traumas et le suicide. Avec un peu de chance, ces données nous permettront d'effectuer une évaluation encore plus nuancée et durable de sujets relatifs à la pandémie, par exemple l'épidémiologie traumatique globale, la préparation systémique aux traumas, le suicide, la violence interpersonnelle intentionnelle (familiale ou non), la santé des femmes et la prévention des crimes par armes à feu. Il sera particulièrement intéressant d'étudier l'influence des variations potentielles entre les expériences sociales, les systèmes de santé et les conditions économiques ${ }^{4}$ des provinces canadiennes sur ces sujets d'étude. On doit aussi s'attendre à ce que certaines interprétations évoluent dans le temps, parallèlement à la COVID-19. Pour accomplir tout cela, que ce soit en période de crise ou de stabilité, nous devrons mettre en place des stratégies de surveillance sophistiquées qui vont au-delà des registres de traumatologie. Si nous voulons être prêts à affronter la prochaine menace mondiale, l'évaluation honnête de la situation et la préparation à l'échelle nationale et locale seront essentielles.

\section{Chad G. Ball, MD, MSc}

Corédacteur en chef, fournal canadien de chirurgie

Intérêts concurrents : None declared.

Propriété intellectuelle du contenu : Il s'agit d'un article en libre accès distribué conformément aux modalités de la licence Creative Commons Attribution (CC BY-NC-ND 4.0), qui permet l'utilisation, la diffusion et la reproduction dans tout médium à la condition que la publication originale soit adéquatement citée, que l'utilisation se fasse à des fins non commerciales (c.-à-d., recherche ou éducation) et qu'aucune modification ni adaptation n'y soit apportée. Voir : https:// creativecommons.org/licenses/by-nc-nd/4.0/deed.fr.

DOI: $10.1503 /$ cjs.007121

\section{References}

1. Harvey EJ. Lessons (so far) from the COVID-19 pandemic. Can $\mathcal{F}$ Surg 2021;64:E108.

2. Abdallah HO, Zhao C, Kaufman E, et al. Increased firearm injury during the COVID-19 pandemic: a hidden urban burden. $7 \mathrm{Am}$ Coll Surg 2021;232:159-68.

3. Ball CG, Das D, Roberts DJ, et al. The evolution of trauma surgery at a high-volume Canadian centre: implications for public health, prevention, clinical care, education and recruitment. Can $\mathcal{F}$ Surg 2015;58:19-23.

4. Roberts DJ, Das D, Mercado M, et al. A booming economy means a bursting trauma system: association between hospital admission for major injury and indicators of economic activity in a large Canadian health region. Am F Surg 2014;207:653-7. 